\title{
Double retromolar foramen during an inferior third molar extraction: a rare case report
}

\author{
Olavo Barbosa de Oliveira-Neto, ${ }^{1}$ Yasmin Lima Nascimento, ${ }^{2}$ Fernando José Camello de Lima, ${ }^{3}$ Célio Fernando de Sousa Rodrigues, ${ }^{3}$ Felippe Bevilacqua \\ Prado, ${ }^{4}$ Ana Cláudia Rossi, ${ }^{4}$ Alexandre Rodrigues Freire ${ }^{4}$ \\ ${ }^{1}$ Department of Anatomy, Federal University of Pernambuco, Recife, Pernambuco, Brazil \\ ${ }^{2}$ School of Dentistry, Federal University of Alagoas, Maceió, Alagoas, Brazil \\ ${ }^{3}$ Department of Morphology, Anatomy Division, Institute of Health and Biological Sciences, Federal University of Alagoas, Maceió, Alagoas, Brazil \\ ${ }^{4}$ Department of Morphology, Anatomy Division, Piracicaba Dental School, University of Campinas, Piracicaba, São Paulo, Brazil \\ - Conflicts of interest: none declared.
}

\section{Abstract}

Objective: the aim of the present study was to report the occurrence of a double retromolar foramen during the extraction of a mandibular third molar. Case report: a 20 -year-old male patient was referred to the dental clinic of the Federal University of Alagoas for extraction of the maxillary and mandibular left third molars due to orthodontic reasons. A double retromolar foramen was identified perioperatively during muco-periosteal detachment on the retromolar trigone region, distally to the mandibular left third molar, whereas there was a mild hemorrhage, which was controlled using gaze soaked in a solution of local anesthetic with vasoconstrictor. The occurrence of this anatomical variant was registered by means of photographs. Prior to the procedure, the patient's panoramic radiograph suggested the presence of the aforementioned anatomical structure. Conclusion: the knowledge regarding the occurrence of this inconstant foramen presents clinical importance for mandibular third molar extractions and can aid the dentist in avoiding and handling accidents and complications during this surgical procedure.

Key words: Retromolar foramen; Retromolar canal, Occurrence, Anatomy, Mandible.

\section{Introduction}

$\mathrm{T}$ The retromolar foramen is the opening of the retromolar canal in the mandible. ${ }^{1}$ It regards an inconstant foramen that occurs distally to the last mandibular molar, on the retromolar trigone or the retromolar fossa regions. It varies in number from one to three and its occurrence might be as high as $91,3 \%{ }^{2}$

This foramen may carry a neurovascular bundle that is associated to the innervation of the tendon of the temporal muscle, of the buccinator muscle, periodontium, and dental pulp of the lower third molar. ${ }^{3}$ Since the ossification of the mandibular body occurs around the inferior alveolar nerve, there is an embryological basis to explain why accessory mandibular canals, such as the retromolar canal, are often associated to having a nerve and/or associated blood vessels in its content. ${ }^{4}$

The presence of this anatomical variant is of clinical importance because it can lead to accidents and complications during surgical procedures in the mandible, such as mandibular third molar extraction and dental implant placement, which may cause paresthesia, severe hemorrhage, or traumatic neuroma. This foramen may also be associated to failures in the inferior alveolar nerve block. ${ }^{5,6}$

Therefore, the aim of the present study was to report the occurrence of a double retromolar foramen during the extraction of a mandibular third molar.

\section{Case report}

A 20-year-old male patient (A.M.N.S) was referred to the dental clinic of the Federal University of Alagoas for extraction of the maxillary and mandibular left third molars due to orthodontic reasons.

During anamnesis, the patient denied the presence of underlying pathologies that could endanger his health and the surgery performance. The physical examination did not show local alterations. The radiograph exam (panoramic radiograph) provided by the patient showed the following (Fig. 1.a): 1) an erupted and slightly extruded maxillary left third molar and an erupted and vertically positioned mandibular left third molar with no bone coverage of the mandibular ramus (Pell and Gregory type 1A);2) the suggestion of close proximity between the distal root of the mandibular left third molar and the inferior alveolar canal; 3) the suggestion of presence of a retromolar canal ending in two retromolar foramens on the retromolar trigone region; 4) the suggestion of presence of an accessory canal that emits the retromolar canal and ends on the apical region and distal surface of the left mandibular third molar.

The patient was lectured regarding the risks of the procedure, such as paresthesia and hemorrhage, and authorized the surgery after having demonstrated understanding regarding the provided explanations. 

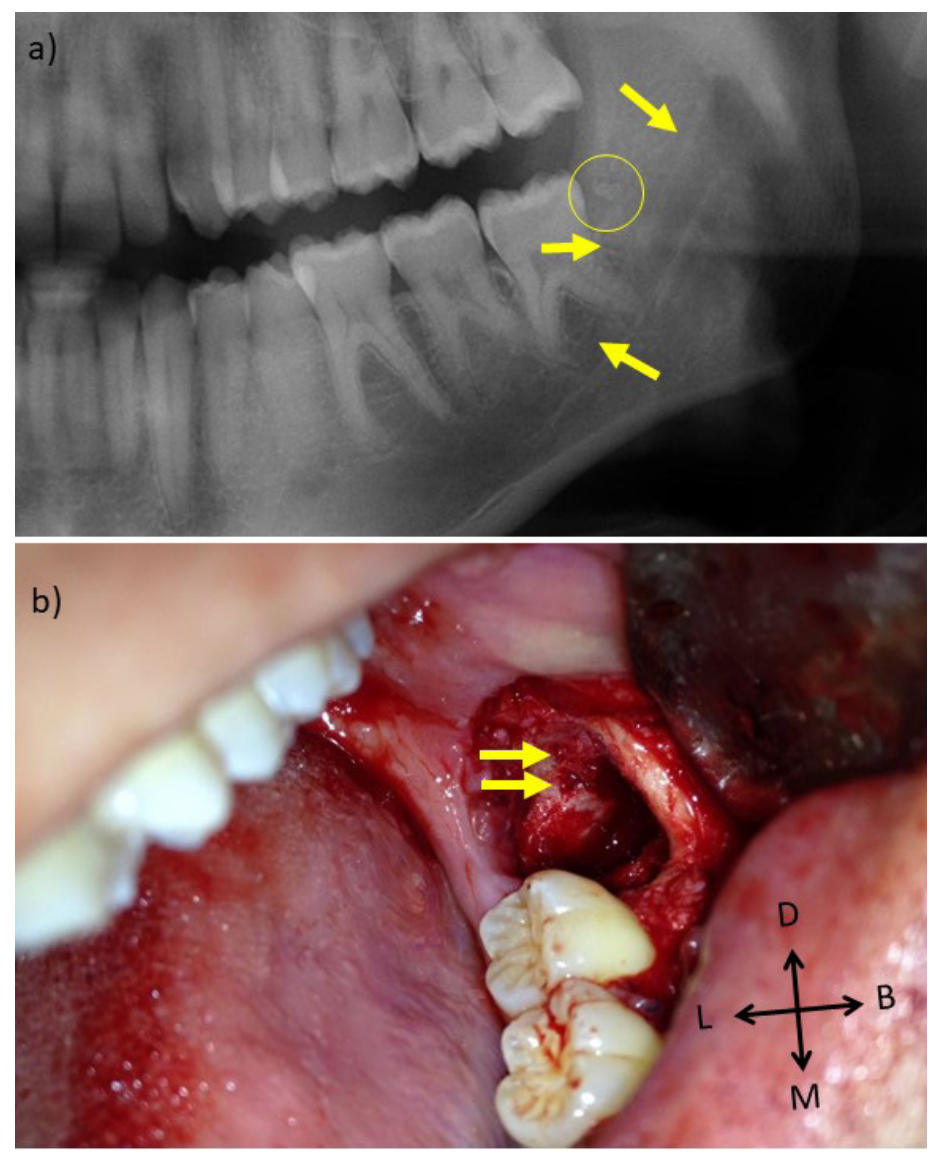

Figure 1. a) Panoramic radiograph of the patient. One can see that the radiograph suggests the presence of a retromolar canal that is emitted from an accessory canal and ends in two foramens (circle) on the left retromolar trigone region. The accessory canal ends on the apical region and distal surface of the left mandibular third molar; b) Perioperative image showing a double retromolar foramen (arrows) on the retromolar trigone region distally to the socket of the extracted mandibular left third molar. Hemostasis was obtained prior to the moment when the photograph was taken.

The patient received four capsules of amoxicillin (500 $\mathrm{mg}$ ) and two pills of dexamethasone (4 $\mathrm{mg})$ one hour before surgery (both taken orally for antibiotic and anti-inflammatory prophylaxis).

Intra-oral antisepsis was performed using $30 \mathrm{ml}$ of a $0,12 \%$ chlorhexidhine gluconate oral rinse (Periogard, Colgate). Extra-oral antisepsis was also performed using a $2 \%$ chlorhexidhine gluconate (Riohex, Rioquímica) on the oral, buccal, and mental regions.

The surgical procedure begun with the superior alveolar and greater palatine nerve blocks for the maxillary left third molar extraction; then, syndesmotomy was performed and the tooth was extracted using Cryer elevators. The region was then sutured with a 4.0 nylon thread and the inferior alveolar, lingual, and buccal nerve blocks were performed for the mandibular left third molar extraction.

A intra-sulcular incision with a distal relaxing incision was made using a number 15 scalpel blade set on a number 3 scalpel, and, after syndesmotomy, a full muco-periosteal flap was raised in the buccal region of the first, second, and third mandibular molars and on the retromolar region as well, during which there was a mild hemorrhage arousing from the retromolar region where two retromolar foramens were identified (Fig. 1.b). There was an attempt to control the hemorrhage with gaze compression, which did not yield success. Hemostasis was only obtained by compressing gaze soaked in a solution of local anesthetic and vasoconstrictor (2\% lidocaine and epinephrine 1:100.000). After hemorrhage control, osteotomy was performed with a number 702 surgical bur set in high rotation and abundantly irrigated with saline, and the tooth was extracted using straight dental elevators. Odontosection was not necessary. The region was also sutured with a 4.0 nylon thread.

The patient was instructed to perform post-operative care and amoxicillin (500mg, 1 capsule, three times a day for 7 days), ibuprofen (600mg, 1 pill, three times a day for 3 days), and paracetamol (750mg, 1 pill, three times a day for 3 days) were prescribed.

Four tubes of anesthetic (2\% lidocaine and epinephrine 1:100.000 - Alphacaine, Nova DFL) were used in total for the extraction of both teeth. It was necessary to complement anesthesia during the extraction of the lower left third molar by means of intraligamentary injection.

Once teeth were extracted, three roots were identified on the lower left mandibular molar, i.e., a mesial root, a distal root, and a supernumerary median root (Fig. 2).

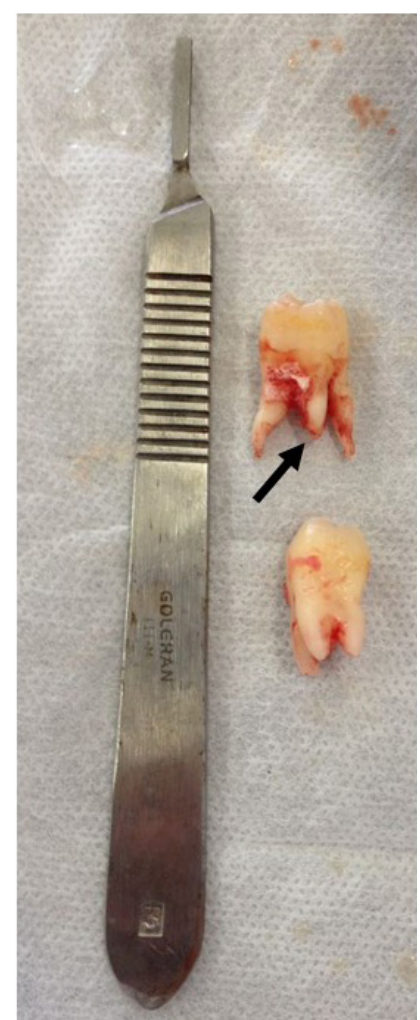

Figure 2. Maxillary and mandibular left third molars after extraction. One can see a median supernumerary root on the mandibular left third molar. 
On the follow-up consultation after seven days of surgery, the patient reported the occurrence of mild edema and trismus during the first five days after surgery; he also reported no pain and no paresthesia on the retromolar region or in regions associated to the innervation of the inferior alveolar nerve.

\section{Discussion}

The present case report describes the occurrence of two retromolar foramens on the retromolar trigone region, which were identified during a lower left third molar extraction.

The occurrence, number, and mandibular region of the retromolar foramen were studied by many authors. ${ }^{1,2,6,7,8,10}$ Regarding its occurrence and number, one, two, and three retromolar foramens can occur, respectively, up to $55.93 \%$, $16.22 \%$, and $8.11 \%$ of mandibles. ${ }^{1}$ The retromolar trigone and retromolar fossa are the preferred regions to identify these structures and black female individuals are more prone to have this anatomic variant. $6,7,10$

Hence, retromolar foramens are well addressed in anatomical scientific literature; however, to the best of the authors' knowledge, there is no report regarding a double retromolar foramen identified during a surgical procedure as well as there is no report of its clinical content from peri and post-operatively examinations. There is one study that reported the occurrence of a triple retromolar foramen, however, this report was of a 3-dimensionally reconstructed mandible that was resected from an 84-year-old cadaver who died from gallbladder cancer. ${ }^{3}$ Therefore, the present case report can be considered as a rare one.

The content of the retromolar canal and foramen was discussed by several authors, which suggested the presence of nerves, blood vessels, or both. In our case report, the perioperative hemorrhage that arouse from the retromolar foramens seemed to be of venous origin, since the blood did not squirt, but ran through the retromolar foramens and soaked the surgical bed. In addition, the patient did not report the occurrence of paresthesia on the retromolar region, which can indicate that the retromolar foramens identified in the present study did not carry a nerve. However, one must highlight that it was necessary to complement the anesthesia using the intraligamentary injection for tooth extraction and the panoramic radiograph showed that the accessory canal continued into the mandible and ended on the apical region and distal surface of the lower left mandibular molar. This might be an indication that this accessory canal carried a nerve to the dental pulp of the extracted tooth and adjacent periodontium.

The anatomical knowledge regarding this mandibular variant can help the dentist in deciding the best surgical approach in this region, as well as in decision-making when this structure is identified during surgical procedures, avoiding injuries to the structures that these foramens carry. Moreover, the aforementioned items can be added together in order to preserve patients' health, in this context.

\section{Conclusion}

The knowledge regarding the occurrence of this inconstant foramen presents clinical importance for mandibular third molar extractions and can aid the dentist in avoiding and handling accidents and complications during this surgical procedure.

\section{Funding}

This study was solely funded by the authors.

\section{References}

1. Rossi AC, Freire AR, Prado GB, Prado FB, Botacin PR, Caria PHF (2012). Incidence of Retromolar Foramen in Human Mandibles: Ethnic and Clinical Aspects. Int. J. Morphol.,30(3):1074-78.

2. Lizio G, Pelliccioni GA, Ghigi G, Fanelli A, Marchetti C (2013). Radiographic assessment of the mandibular retromolar canal using cone-beam computed tomography. Acta Odontol Scand; 71: 650-55.

3. He P, Iwanaga J, Truong M K, et al. First Report of Tripled Retromolar Foramina (2017). Cureus 9(7): e1440.

4. Lima FJC, Oliveira Neto, OB, Barbosa FT, Dantas LCS, Olave E, Sousa-Rodrigues CF (2016). Occurrence of the Accessory Foramina of the Mandibular Ramus in Brazilian Adults and its Relation to Important Mandibular Landmarks. Int. J. Morphol., 34(1):330-34.

5. Potu BK, Jagadeesan S, Bhat KMR, Sirasanagandla SR. Retromolar foramen and canal: A comprehensive review on its anatomy and clinical applications (2013). Morphologie, 97: 31-37.
6. Gamieldien MY, Van Schoor A. Retromolar foramen: an anatomical study with clinical considerations (2013). Br J Oral Maxillofac Surg.;54(7):784-7. 7. Alves N, Deana NF. Anatomical and radiographical study of the retromolar canal and retromolar foramen in macerated mandibles (2015). Int J Clin Exp Med;8(3):4292-96.

8. Kikuta S, Iwanaga J, Nakamura K, Hino K, Nakamura M, Kusukawa J (2018). The retromolar canals and foramina: radiographic observation and application to oral surgery. Surg Radiol Anat 40(6):647-52.

9. Truong MK, He P, Adeeb N, Oskouian RJ, Tubbs RS, Iwanaga J. Clinical Anatomy and Significance of the Retromolar Foramina and Their Canals: A Literature Review (2017). Cureus., 17;9(10):e1781.

10. Capote TSO, Gonçalves MA, Campos JADB. Retromolar Canal Associated with Age, Side, Sex, Bifid Mandibular Canal, and Accessory Mental Foramen in Panoramic Radiographs of Brazilians (2015). Anat Res Int.;2015: 434083. 


\section{Mini Curriculum and Author's Contribution}

1. Olavo Barbosa de Oliveira Neto - DDS; MSc. Contribution: performed the surgical procedure, wrote and submitted the manuscript. ORCID: 0000-0003-1280-659X 2. Yasmin Lima Nascimento - Graduate dentist. Contribution: assisted during surgery and took the perioperative photographs used in this manuscript. ORCID: 0000-0002-8643-1535

3. Fernando José Camello de Lima - DDS; PhD. Contribution: provided clinical knowledge to the anatomical findings. ORCID: 0000-0003-1593-7747

4. Célio Fernando de Sousa Rodrigues - MD; PhD. Contribution: provided insights to the article's structure. ORCID: 0000-0002-1361-8139

5. Felippe Bevilacqua Prado - DDS; PhD. Contribution: reviewed the first draft of the manuscript. ORCID: 0000-0001-5636-6915

6. Ana Cláudia Rossi - DDS; PhD. Contribution: provided insights to the article's structure and reviewed the first draft of the manuscript. ORCID: 0000-0002-87184898

7. Alexandre Rodrigues Freire - DDS; PhD. Contribution: reviewed and approved the final version of the manuscript. ORCID: 0000-0002-0175-0896

Submitted: 10/02/2019 / Accepted for publication: 11/06/2019

Corresponding author

Olavo Barbosa de Oliveira Neto

E-mail: olavobarbosa91@gmail.com 